\title{
Correlation between dielectric constant and chemical structure of sodium silicate glasses
}

\author{
C. H. Hsieh and H. Jain a) \\ Department of Materials Science and Engineering, 5 East Packer Avenue, Lehigh University, Bethlehem, \\ Pennsylvania 18015
}

\author{
E. I. Kamitsos \\ Theoretical and Physical Chemistry Institute, National Hellenic Research Foundation, 48 Vass. \\ Constantinou Avenue, Athens 116 35, Greece
}

(Received 18 September 1995; accepted for publication 30 April 1996)

\begin{abstract}
The chemical structure of sodium aluminosilicate glasses is determined by high resolution x-ray photoelectron spectroscopy (XPS) as silicon is gradually replaced by aluminum. A well-defined chemical state is found for silicon, aluminum, and sodium atoms, while three different environments are identified for oxygen atoms corresponding to $\mathrm{Si}-\mathrm{O}-\mathrm{Si}, \mathrm{Si}-\mathrm{O}-\mathrm{Al}$, and $\mathrm{Si}-\mathrm{O}-\mathrm{Na}$ bonds. The binding energy of $\mathrm{Na} 1 s$ photoelectrons increases significantly with increasing aluminum substitution while that of $\mathrm{Al} 2 p$ and components of $\mathrm{O} 1 s$ photoelectrons remains approximately constant. Thus, the ionicity of sodium increases with aluminum amount, but the over all electron density around silicon, aluminum, and different types of oxygen atoms remains unchanged. The dielectric constant of the glasses increases with increasing aluminum substitution. It is analyzed in terms of the polarizabilities of constituent structural units, viz., silicon tetrahedra, nonbridging oxygen-sodium ion pairs, and aluminum tetrahedron-sodium ion pairs. The electronic polarizability of oxygen ions depends linearly on their negative charge and can be correlated to the $\mathrm{O} 1 s$ XPS binding energy. The ionic polarizability of sodium ions increases with an increasing aluminum amount, and correlates directly with the Na $1 s$ XPS binding energy. (C) 1996 American Institute of Physics. [S0021-8979(96)06015-X]
\end{abstract}

\section{INTRODUCTION}

Dielectric constant is a property of glass which is particularly important for many applications in electronics. For example, glass with low dielectric constant $(<10)$ is a crucial element of high-performance microelectronic systems. It is used as a substrate or a passivation and dielectric layer in semiconductor packaging, ${ }^{1-3}$ and in thick film resistors. ${ }^{4}$ Glasses for such applications include silica and borosilicateand cordierite-based compositions. ${ }^{2,5,6}$ On the other hand, a glass with high dielectric constant is desirable for applications such as high energy capacitors ${ }^{7}$ and multilayer dielectrics. ${ }^{4}$ Typically, high dielectric constant glasses contain heavy-metal cations like $\mathrm{Pb}, \mathrm{Ba}, \mathrm{Bi}$, and $\mathrm{W}{ }^{7}$

To tailor the dielectric constant of glass for a certain application, it is important to understand the mechanism of dielectric polarization which arises from the constituent species including ions, ion pairs, and other building units of the structure. Typically the dielectric constant is analyzed in terms of the polarizability of the ions or structural units. For example, Kenmuir et al. ${ }^{8}$ have studied $\mathrm{Mg}-\mathrm{Al}-\mathrm{Si}$ oxynitride glasses and found that the addition of nitrogen increases the dielectric constant. Singer and Tomozawa ${ }^{5}$ have found for cordierite-based oxyfluoride glasses that fluorine substitution results in a lower dielectric constant. They conclude that, along with the reduced number of polarizable ions per unit volume, the lower polarizability of fluorine than that of oxygen ions reduces the dielectric constant. For the $\mathrm{PbO}-\mathrm{B}_{2} \mathrm{O}_{3}-\mathrm{SiO}_{2}-\mathrm{GeO}_{2}$ glasses, Kobayashi ${ }^{3}$ has suggested

\footnotetext{
a) Author to whom correspondence should be addressed; Electronic mail: hj00@lehigh.edu
}

that the substitution of highly polarizable $\mathrm{Pb}^{2+}$ ions by less polarizable $\mathrm{B}^{3+}$ or $\mathrm{Si}^{4+}$ ions decreases the overall polarizability and increases the stability of glasses. Hampton et al. ${ }^{9}$ and El-Mallawany ${ }^{10}$ have explained the dielectric constant of pure and binary tellurite glasses by considering the polarizability of $\mathrm{TeO}_{2}$ and $\mathrm{WO}_{3}$ units. They report that the polarizability is higher for a $\mathrm{TeO}_{2}$ than a $\mathrm{TeO}_{2}-\mathrm{WO}_{3}$ unit. Sidek et al. ${ }^{11}$ have found for the samarium phosphate glasses that $\mathrm{Sm}_{2} \mathrm{O}_{3}$ does not alter the polarizability at low concentrations and that the dielectric constant is determined by the $\mathrm{P}_{2} \mathrm{O}_{5}$ polarizability. Often, the polarizability has been considered independent of composition. For example, in the study of cordierite-based oxyfluoride glasses, ${ }^{5}$ the same value of polarizability has been assigned for oxygen and fluorine ions independent of composition. On the other hand, sometimes the polarizability is considered to be dependent on composition, such as, in the study of pure and binary tellurite glasses, ${ }^{10}$ where the decrease of polarizability from $\mathrm{TeO}_{2}$ to $\mathrm{TeO}_{2}-\mathrm{WO}_{3}$ units is explained by the increase in $\mathrm{Te}-\mathrm{O}$ and $\mathrm{W}-\mathrm{O}$ bond energies.

In general, one expects that the polarizability of ions and structural units would depend on the charge and bond strength of the polarizable species. However, to the best of our knowledge an explicit correlation between the dielectric constant and chemical structure has never been established. In our previous study of the structure of sodium aluminosilicate (SAS) glasses by $\mathrm{x}$-ray photoelectron spectroscopy (XPS) ${ }^{12}$ it was found that sodium ions are only partially ionized in sodium trisilicate glass and their ionicity increases when silicon is substituted by aluminum. The physical structure of the glass remains essentially unaffected because the 

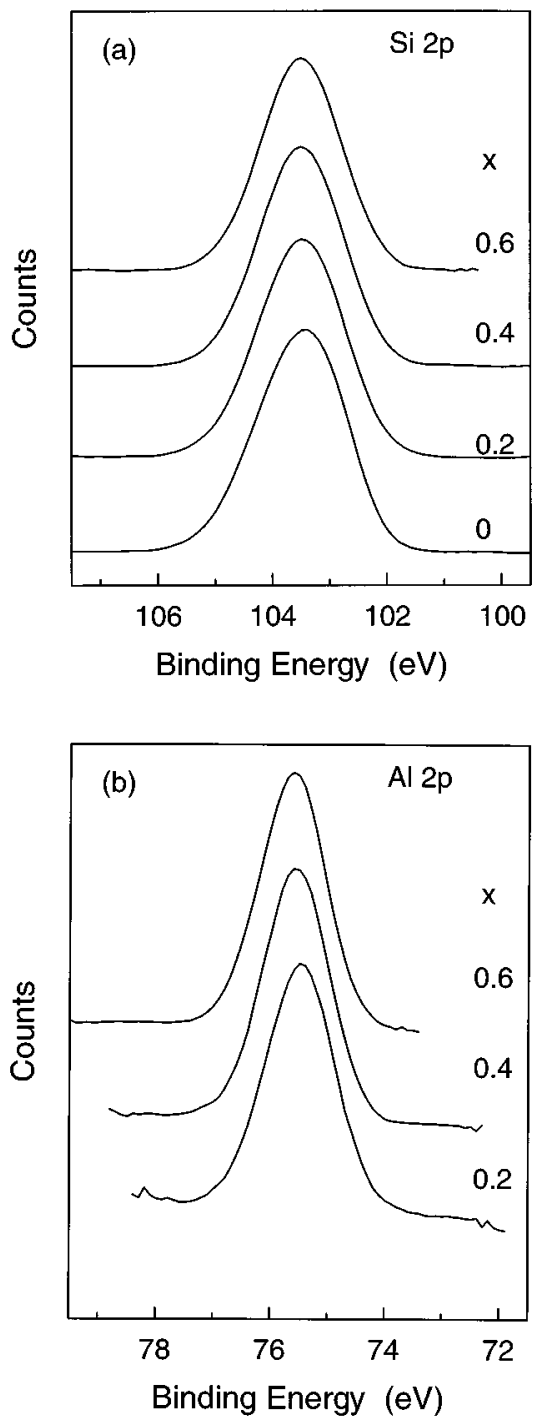
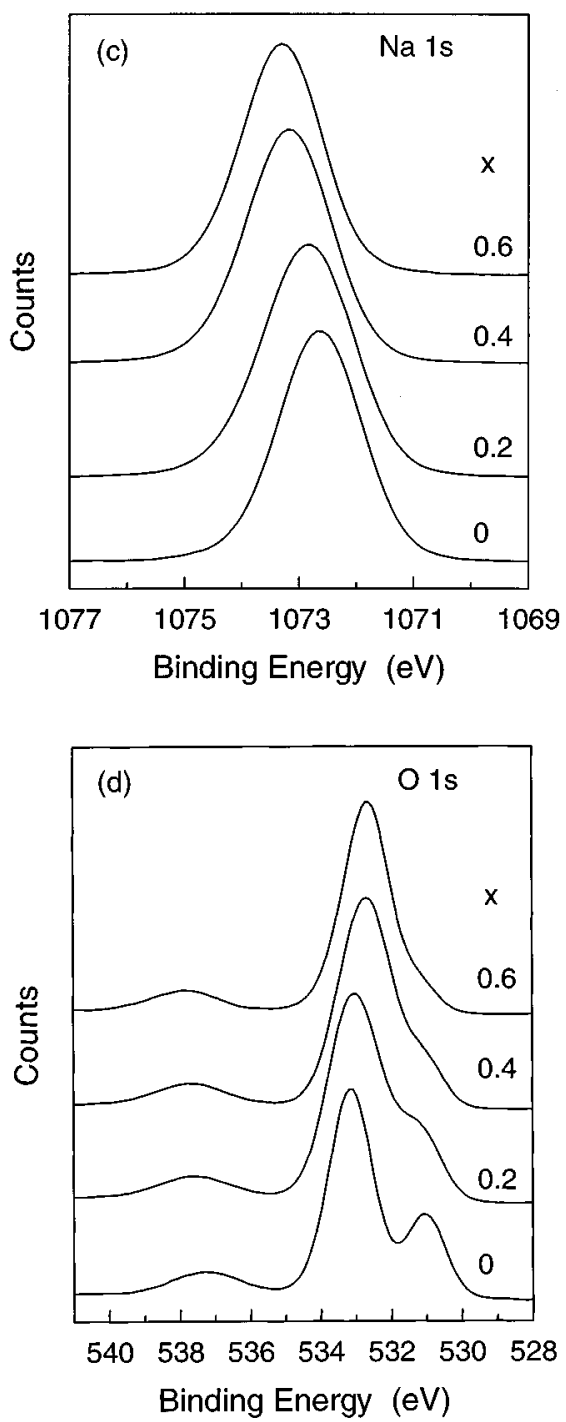

FIG. 1. X-ray photoelectron spectra of (a) Si $2 p$, (b) Al $2 p$, (c) Na $1 s$, and (d) $\mathrm{O} 1 s$ for the SAS glasses.

basic building block of the structure, $\mathrm{SiO}_{4}$ tetrahedron, is simply replaced by an $\mathrm{AlO}_{4}$ tetrahedron without appreciably affecting the molar volume. These observations offer a model example for understanding how the redistribution of electrons and a change of chemical structure can affect the dielectric constant of glasses. Thus, the purpose of this article is: first, to present additional details of XPS for understanding all the bonding changes in the structure of sodium aluminosilicate glass; second, to investigate the polarizability of ions or structural units; and finally, to discuss the correlation between dielectric constant and chemical structure.

\section{EXPERIMENT}

\section{A. Sample preparation}

The glasses were prepared according to the formula $\mathrm{Na}_{2} \mathrm{O} x \mathrm{Al}_{2} \mathrm{O}_{3}(3-2 x) \mathrm{SiO}_{2}$ where $x=0,0.2,0.4$, and 0.6. This composition series emphasizes the substitution of silicon by aluminum so that the ratio of the concentration of network modifier $(\mathrm{Na})$ to network former $(\mathrm{Si}+\mathrm{Al})$ cations remained constant. The glasses were made from reagent grade
$\mathrm{Na}_{2} \mathrm{CO}_{3}, \mathrm{Al}_{2} \mathrm{O}_{3}$, and $\mathrm{SiO}_{2}$ by conventional melt-quench method. The annealed glass samples were homogeneous and showed no stress when viewed with polarized light. The samples were cut to $1 \mathrm{~mm}$ thickness for further experiments. For details of glass preparation, the reader is referred to Ref. 12.

\section{B. X-ray photoelectron spectroscopy}

The Si $2 p$, Na $1 s, \mathrm{O} 1 s$, and $\mathrm{Al} 2 p$ x-ray photoelectron spectra were obtained using a high resolution Scienta ESCA300 spectrometer. The instrument was operated in a mode that yielded a Fermi level width of $0.4 \mathrm{eV}$ for silver. At this level of resolution, the instrumental contribution to the line width was extremely small. The spectra were taken on a freshly created sample surface obtained by fracturing it in situ inside the high vacuum chamber. The sample surface was flooded with a beam of low energy electrons to minimize the surface charging. Further details of the experiment are given elsewhere. ${ }^{12}$ 


\section{Dielectric constant measurement}

The dielectric constant was measured using a General Radio 1621 capacitance measurement system which utilizes a Wheatstone-type capacitance bridge with transformer ratio arms. Gold electrodes with a three-terminal configuration were applied to the sample surfaces by vacuum evaporation. The sample was placed in a tube furnace, and the temperature was monitored by a thermocouple which almost touched the sample. The sample cell and all leads were surrounded by a shield connected to ground to avoid electromagnetic interference. The ac capacitance $C$ and conductance $G$, of the samples were determined by balancing the capacitance bridge in the frequency $f$ range of $10 \mathrm{~Hz}-100 \mathrm{kHz}$ at room temperature. Dielectric constant, $\epsilon(f)$, was calculated from capacitance by multiplying with an appropriate geometric factor.

\section{Density measurement}

The density was measured at room temperature using the Archimedes method with deionized water with an error $<0.1 \%$.

\section{RESULTS}

\section{A. X-ray photoelectron spectroscopy}

The photoelectron spectra for Si $2 p$, Al $2 p$, and $\mathrm{Na} 1 s$ electrons show a symmetric single peak for the present SAS glasses [Figs. 1(a)-1(c)]. The spectra for $\mathrm{O} 1 s$ electrons, however, reveal in Fig. 1(d) a second peak at the lower binding energy side of the main peak for sodium trisilicate glass, which becomes gradually smaller, ultimately appearing as a shoulder of the main peak when the substitution by $\mathrm{Al}$ increases. This result was explained by considering the presence of three distinct types of oxygen atoms in $\mathrm{Si}-\mathrm{O}-\mathrm{Si}$, $\mathrm{Si}-\mathrm{O}-\mathrm{Al}$, and $\mathrm{Si}-\mathrm{O}-\mathrm{Na}$ bonds. ${ }^{12}$ Here the $\mathrm{O} 1 s$ spectra are decomposed into three components by first choosing the relative charge density of various types of oxygen as a guide for the initial binding energies for the three components. The computer program then determines the best fitted parameters with the constraint that the peak positions remain within $\pm 0.5 \mathrm{eV}$. Under these conditions, the error bar is $\sim 0.1 \mathrm{eV}$ for the various oxygen peaks, which is the same as the experimental error. For evaluating the various spectra, the $\mathrm{Si}$ $2 p$ peak at $103.5 \mathrm{eV}$ is chosen as the most appropriate internal binding energy reference because the chemical environment of silicon remains essentially unaffected by aluminum substitution. ${ }^{12}$ The binding energies of $\mathrm{Na} 1 s, \mathrm{Al} 2 p$, and $\mathrm{O}$ $1 s$ peaks for the three types of oxygen ions are reported in Table I. It can be seen from Table I that, while the binding energies of A1 $2 p$ and $\mathrm{O} 1 s$ electrons do not change significantly, the binding energy of $\mathrm{Na} 1 s$ electrons increases by $\sim 0.7 \mathrm{eV}$ from sodium trisilicate glass to the glass containing the largest amount of aluminum.

\section{B. Dielectric constant}

The measured dielectric constants of the four glasses at $25 \pm 0.7^{\circ} \mathrm{C}$ are reported as a function of frequency in Fig. 2. It shows that the dielectric constant decreases for each glass
TABLE I. Binding energy (eV) of Na $1 s, \mathrm{O} 1 s$, and $\mathrm{Al} 2 p$ electrons of the SAS glasses.

\begin{tabular}{lccccc}
\hline \hline & & \multicolumn{4}{c}{$\mathrm{O} 1 s$} \\
\cline { 3 - 5 } $\mathrm{Al} / \mathrm{Na}$ & $\mathrm{Na} 1 s$ & $\mathrm{Si}-\mathrm{O}-\mathrm{Si}$ & $\mathrm{Si}-\mathrm{O}-\mathrm{Al}$ & $\mathrm{Si}-\mathrm{O}-\mathrm{Na}$ & \multirow{2}{*}{$\mathrm{Al} 2 p$} \\
\hline 0 & $1072.6(4)$ & 533.1 & $\ldots$ & 531.0 & $\ldots$ \\
0.2 & $1072.8(5)$ & 533.2 & 532.6 & 531.2 & 75.5 \\
0.4 & $1073.1(9)$ & 533.2 & 532.5 & 531.1 & 75.6 \\
0.6 & $1073.3(1)$ & 533.5 & 532.6 & 531.3 & 75.6 \\
\hline \hline
\end{tabular}

when the frequency increases. To obtain the dielectric constant that arises only from the electronic and ionic polarizations, the data are analyzed using Cole-Cole plot for complex dielectric constant $\epsilon^{*}=\epsilon^{\prime}-i \epsilon^{\prime \prime}$, where $\epsilon^{\prime}$ is the real part, and $\epsilon^{\prime \prime}$ is the imaginary part of the dielectric constant. $\epsilon^{\prime}$ is equal to the measured $\epsilon$, and $\epsilon^{\prime \prime}=\sigma / \omega \epsilon_{0}$ where $\sigma$ is the conductivity, $\omega$ is the angular frequency, and $\epsilon_{0}$ is the vacuum permittivity. A Cole-Cole plot is a complex dielectric constant plot with $\epsilon^{\prime}$ on the $x$-axis and $\epsilon^{\prime \prime}$ on the $y$-axis to show the dielectric relaxation behavior of a material. It is known empirically ${ }^{13}$ that the conductivity of glass follows a power law at high frequencies: $\sigma=\sigma_{\mathrm{dc}}+A \omega^{s}$, where $\sigma_{\mathrm{dc}}$ is the frequency independent dc conductivity, and $A$ and $s(0<s<1)$ are fitting parameters. We identify the frequency dependent component of total conductivity with $\sigma_{\mathrm{ac}} \simeq A \omega^{s}$. Therefore, one has $\epsilon^{\prime \prime}=\epsilon_{\mathrm{dc}}^{\prime \prime}+\epsilon_{\mathrm{ac}}^{\prime \prime}$, where $\epsilon_{\mathrm{dc}}^{\prime \prime}=\sigma_{\mathrm{dc}} / \omega \epsilon_{0}$ and $\epsilon_{\mathrm{ac}}^{\prime \prime}$ $=\sigma_{\mathrm{ac}} / \omega \epsilon_{0}=A / \omega^{1-s} \epsilon_{0}$. Since a Cole-Cole plot is to represent the dielectric relaxation, $\epsilon_{\mathrm{ac}}^{\prime \prime}$ is used in the plot. According to the Kramers-Kronig transformation which relates $\epsilon^{\prime}$ and $\epsilon_{\mathrm{ac}}^{\prime \prime}$ at the high frequency end, the Cole-Cole plot becomes a straight line inclined at an angle $(1-s) \pi / 2$ to the real axis,${ }^{13}$ as shown in Fig. 3 for the SAS glasses. Here $\sigma_{\text {ac }}$ was calculated by subtracting $\sigma_{\mathrm{dc}}$ from the measured $\sigma ; \sigma_{\mathrm{dc}}$ itself was determined by the complex impedance analysis. ${ }^{14}$ The dielectric constant obtained by extrapolating each line to the real axis is shown in Fig. 4. It is called the intermediate-

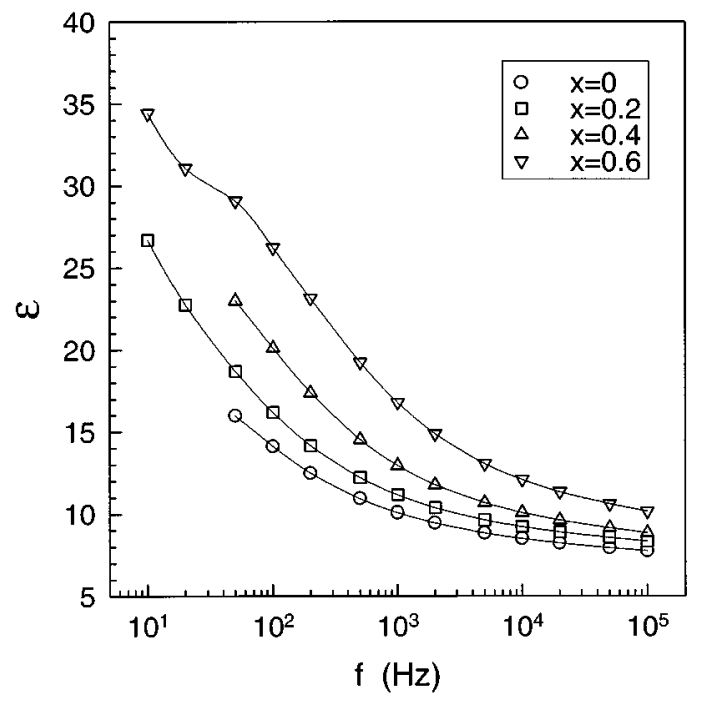

FIG. 2. Frequency dependence of the dielectric constant of the SAS glasses at $25^{\circ} \mathrm{C}$. 


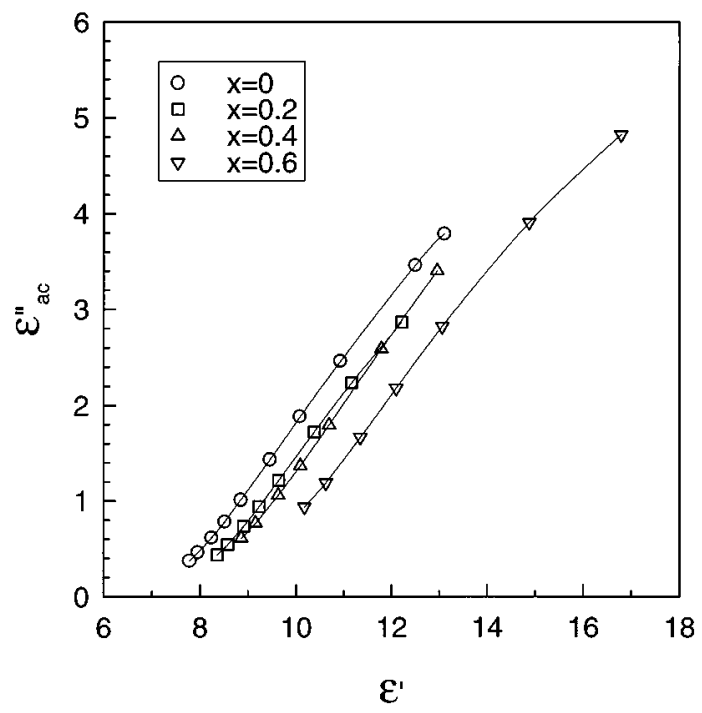

FIG. 3. Cole-Cole plots of the SAS glasses at $25^{\circ} \mathrm{C}$.

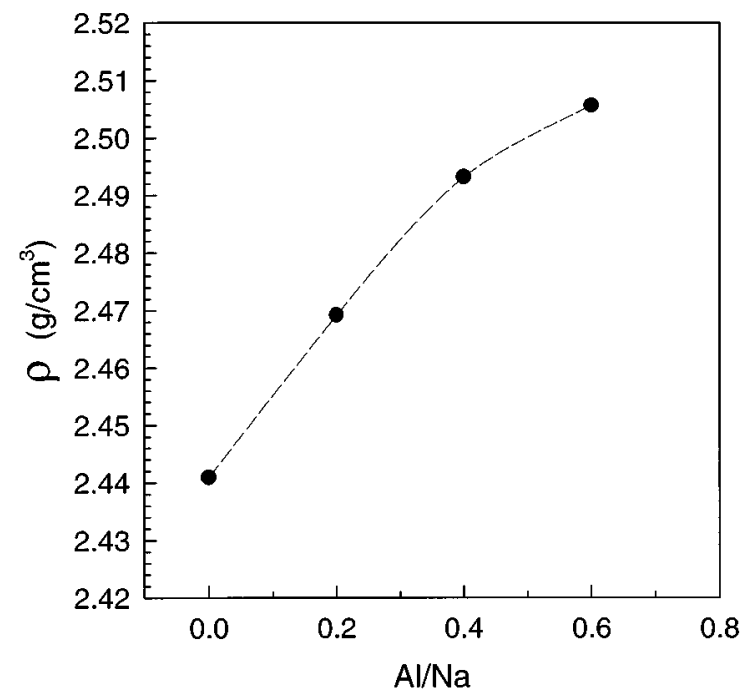

FIG. 5. Densities of the SAS glasses at room temperature. frequency dielectric constant $\epsilon_{\text {i.f. }}$ to distinguish it from the optical-frequency dielectric constant $\epsilon_{\infty}$ which usually stands for the dielectric constant from electronic polarization only. It can be seen from Fig. 4 that $\epsilon_{\text {i.f. }}$ increases with the aluminum content in the glass.

\section{Density}

The composition dependence of the measured densities is shown in Fig. 5. It shows that the density increases monotonically with an increasing amount of Al substitution in the SAS glasses.

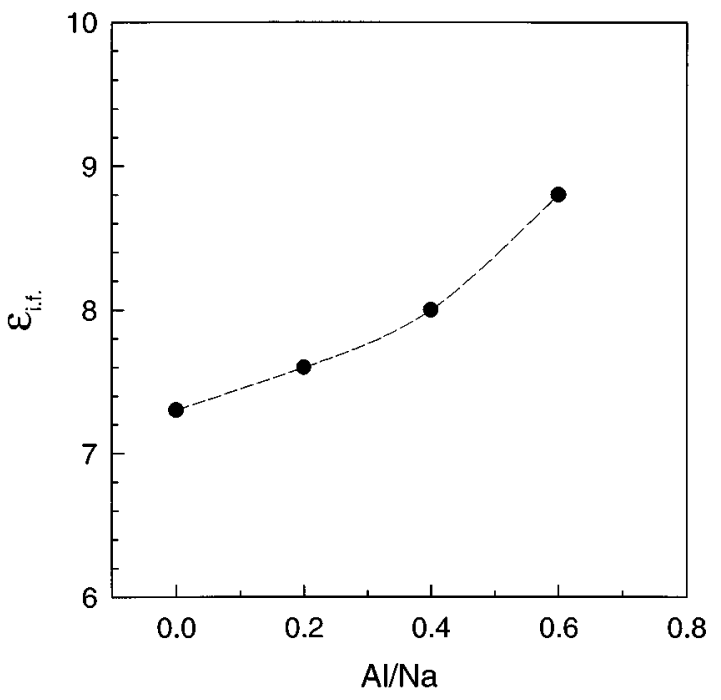

FIG. 4. Intermediate-frequency dielectric constant of the SAS glasses at $25^{\circ} \mathrm{C}$.

\section{DISCUSSION}

\section{A. Chemical structure of SAS glasses}

In the previous XPS study of the SAS glasses, ${ }^{12}$ it was found from the analysis of $\mathrm{O} 1 s$ spectra that aluminum ions form four coordinated tetrahedra at the expense of nonbridging oxygen ions. Since the aluminum tetrahedra, like the nonbridging oxygen ions, have a formal negative charge on them, some of the sodium ions which were associated with the nonbridging oxygen ions in the sodium trisilicate glass would become associated with the aluminum tetrahedra. From the increase of $\mathrm{Na} 1 s$ binding energy, it was concluded that sodium ions are only partially ionized in the sodium trisilicate glass, and their ionicity increases with increasing amount of aluminum substitution. However, it was not clear at that time how the electrons donated by the sodium ions were distributed in the glass. We intend to answer this question in this article and present additional analysis of the chemical bonding and charge distribution in the SAS glasses.

To obtain a comprehensive picture of the chemical structure, it is important to first understand the distribution of $\mathrm{Na}$ $3 s^{1}$ valence electrons in the glasses. When sodium ions are associated with nonbridging oxygen ions, as shown in Fig. 6, the $\mathrm{Na} 3 s^{1}$ electrons participate with $\mathrm{O} 2 p$ electrons in the formation of a bond between the two atoms. The nature of these bonds is only partially ionic, ${ }^{12}$ i.e., the $\mathrm{Na} 3 s^{1}$ electrons are distributed between the nonbridging oxygen and the sodium ions. On the other hand, when sodium ions are associated with aluminum tetrahedra, the $\mathrm{Na} 3 s^{1}$ electrons are donated to the aluminum tetrahedra so that aluminum atoms may form tetrahedral bonds. The $\mathrm{Na} 3 s^{1}$ electrons are therefore more removed from sodium atoms in this case than in the previous case. Accordingly, it is expected that the sodium ions which are associated with aluminum tetrahedra should have a higher ionicity than those associated with nonbridging oxygen ions.

If it was possible to distinguish the sodium ions associated with two different kinds of charge-compensating centers in X-ray photoelectron spectra because of their charge density 
(a)

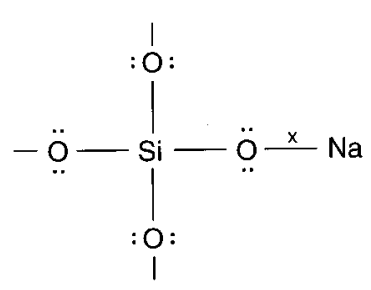

(b)

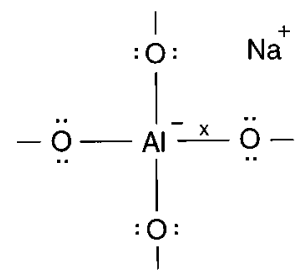

FIG. 6. Lewis electron-dot formulas of (a) a silicon tetrahedron with a nonbridging oxygen and a sodium ion; (b) an aluminum tetrahedron with a sodium ion. The $\mathrm{Na} 3 s^{1}$ electrons are explicitly indicated by the mark $x$.

difference, one would expect two $\mathrm{Na} 1 s$ peaks in the spectra: one with lower binding energy representing the sodium ions associated with nonbridging oxygen ions, and the other with higher binding energy representing the sodium ions associated with aluminum tetrahedra. Furthermore, the relative area ratio of the higher binding-energy peak to the lower one should increase to reflect the increasing aluminum substitution for silicon in the present SAS glass series. However, the experimental results show that there is only one $\mathrm{Na} 1 \mathrm{~s}$ photoelectron peak which shifts to a higher binding-energy position with increasing aluminum substitution. This result indicates that as far as XPS can perceive sodium ions have only one well-defined average chemical state in the SAS glasses instead of having two distinct chemical states corresponding to its bonding with either nonbridging oxygen ions or aluminum tetrahedra.

In recent studies of the structure of sodium aluminosilicate glass by extended x-ray absorption fine structure $(\text { EXAFS })^{15}$ and molecular dynamic (MD) simulation, ${ }^{16,17}$ it is found that the oxygen coordination number of sodium ions is $\sim 5-8$. This coordination number would include all of the three types of oxygen that can be distinguished by XPS, viz., $\mathrm{Si}-\mathrm{O}-\mathrm{Si}$ (bridging oxygen), $\mathrm{Si}-\mathrm{O}-\mathrm{Al}$ (in aluminum tetrahedron), and $\mathrm{Si}-\mathrm{O}-\mathrm{Na}$ (nonbridging oxygen). A sodium ion is, therefore, charge compensated by nonbridging oxygen ions and aluminum tetrahedra at the same time rather than being associated with just one of them. This is illustrated schematically in Fig. 7(a) for a sodium ion surrounded by nonbridging oxygen ions (for charge compensation) and bridging oxygen atoms representing the sodium trisilicate glass; for the case of the SAS glasses, a sodium ion is shown in Fig. 7(b) to be surrounded by both nonbridging oxygen ions and aluminum tetrahedra for charge compensation, and bridging oxygen atoms. Furthermore, since the oxygen coordination number of sodium ion is high, it is reasonable to think that the sodium coordination number of nonbridging oxygen or aluminum tetrahedron is also more than one in the present SAS glasses. It is, therefore, not possible to identify (a)

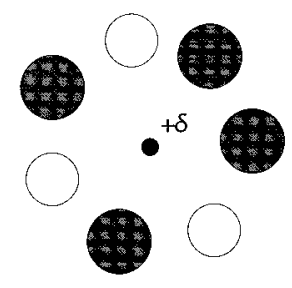

(b)
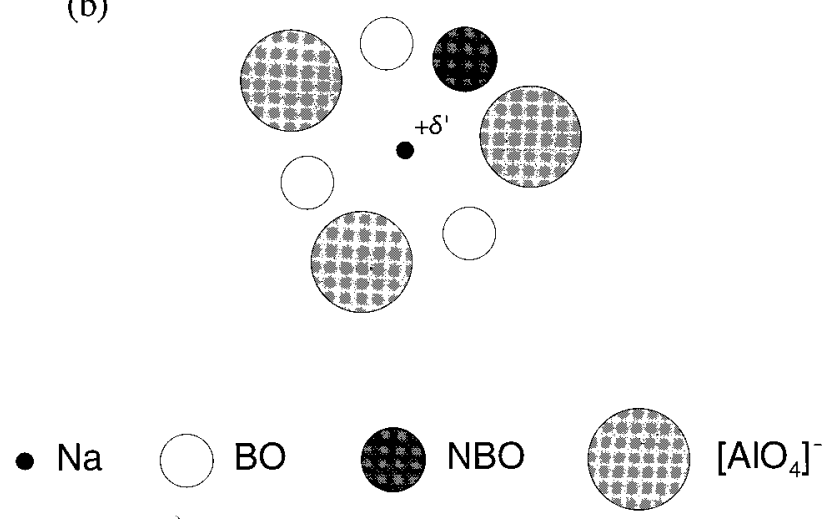

FIG. 7. Environment of a sodium ion consisting of: (a) bridging and nonbridging oxygen atoms in sodium trisilicate glass, and (b) bridging and nonbridging oxygen atoms as well as aluminum tetrahedra in SAS glass. $\delta$ and $\delta^{\prime}$ are charges on sodium ions in respective glasses with $\delta^{\prime}>\delta$.

which sodium ion donates its $3 s^{1}$ electron to a particular nonbridging oxygen or aluminum tetrahedron. It appears more realistic that the sodium ions contribute electrons as a group, and the nonbridging oxygen and the aluminum tetrahedron take the electrons according to their need. Apparently, the electron need is greater for an aluminum tetrahedron than for a nonbridging oxygen. Accordingly the average electron density on each sodium ion decreases when more aluminum atoms substitute in the glass structure.

An important feature of the above model is that with varying aluminum substitution, there is no appreciable change in electron density around aluminum and the three types of oxygen ions; only the amounts of these species change. This is consistent with the XPS results that the binding energies of $\mathrm{Al} 2 p$ and the three $\mathrm{O} 1 s$ peaks do not vary significantly with composition.

In summary, sodium ions have a well-defined chemical state in the SAS glasses. They are only partially ionized in sodium trisilicate glass, and their ionicity increases when silicon is substituted by aluminum. A sodium ion is surrounded by a number of nonbridging oxygen ions and aluminum tetrahedra, and it is not possible to distinguish between the sodium ions which contribute the electron to a certain nonbridging oxygen ion or aluminum tetrahedron. The XPS binding energy of the inner electrons of silicon, aluminum, and oxygen ions remains essentially unchanged.

\section{B. Dielectric constant of SAS glasses}

Dielectric constant of a material originates from the electrical polarization of atoms, ion pairs, or molecular units at a 
TABLE II. Analysis of the dielectric constant of the SAS glasses. $\epsilon_{\text {i.f. }}$ is the intermediate-frequency dielectric constant, $\epsilon_{\infty}$ is the high-frequency dielectric constant, $\rho$ is the density, $V_{m}$ is the molar volume, $P_{m}$ is the molar polarizability, $\alpha_{t}$ is the total molecular polarizability, $\alpha_{j}$ is the polarizability of the $j$ th unit, where unit 1,2 , and 3 are $\left[\mathrm{SiO}_{2}\right],\left[\mathrm{O}_{1 / 2}-\mathrm{Na}\right]$, and $\left[\mathrm{AlO}_{2}-\mathrm{Na}\right]$, respectively. $\Delta \alpha_{t}$ is the difference of total molecular polarizability between a SAS glass and sodium trisilicate glass, and $\Delta \alpha_{j}$ is the polarizability difference between unit 3 and units 1 and 2 . The superscripts $e$ and $i$ refer to the electronic and ionic contributions, respectively.

\begin{tabular}{|c|c|c|c|c|c|c|c|c|}
\hline \multirow[b]{2}{*}{$\mathrm{Al} / \mathrm{Na}$} & \multirow[b]{2}{*}{$\epsilon_{\text {i.f. }}$} & \multirow[b]{2}{*}{$\begin{array}{c}\rho \\
\left(\mathrm{g} / \mathrm{cm}^{3}\right)\end{array}$} & \multirow[b]{2}{*}{$\begin{array}{c}V_{m} \\
\left(\mathrm{~cm}^{3}\right)\end{array}$} & \multicolumn{5}{|c|}{ Total polarizability } \\
\hline & & & & $\begin{array}{c}P_{m} \\
\left(\mathrm{~cm}^{3}\right)\end{array}$ & $\begin{array}{c}\alpha_{t} \\
\left(\AA^{3}\right)\end{array}$ & $\begin{array}{c}\alpha_{j} \\
\left(\AA^{3}\right)\end{array}$ & $\begin{array}{l}\Delta \alpha_{t} \\
\left(\AA^{3}\right)\end{array}$ & $\begin{array}{l}\Delta \alpha_{j} \\
\left(\AA^{3}\right)\end{array}$ \\
\hline 0 & 7.3 & 2.441 & 24.81 & 16.81 & 6.66 & $\alpha_{2}: 5.48$ & $\cdots$ & $\cdots$ \\
\hline 0.2 & 7.6 & 2.469 & 25.43 & 17.48 & 6.93 & $\alpha_{3}: 9.92$ & 0.27 & -0.79 \\
\hline 0.4 & 8.0 & 2.493 & 26.17 & 18.32 & 7.26 & $\alpha_{3}: 10.09$ & 0.60 & -0.62 \\
\hline 0.6 & 8.8 & 2.506 & 27.15 & 19.61 & 7.77 & $\alpha_{3}: 10.53$ & 1.11 & -0.18 \\
\hline \multirow{2}{*}{$\begin{array}{l}\text { Fused } \\
\text { silica }\end{array}$} & 3.8 & 2.20 & 27.31 & 13.18 & 5.23 & $\alpha_{1}: 5.23$ & & \\
\hline & & & & \multicolumn{5}{|c|}{ Electronic polarizability } \\
\hline $\mathrm{Al} / \mathrm{Na}$ & $\epsilon_{\infty}$ & & & $\begin{array}{c}P_{m}^{e} \\
\left(\mathrm{~cm}^{3}\right)\end{array}$ & $\begin{array}{c}\alpha_{t}^{e} \\
\left(\AA^{3}\right)\end{array}$ & $\begin{array}{c}\alpha_{j}^{e} \\
\left(\AA^{3}\right)\end{array}$ & $\begin{array}{l}\Delta \alpha_{t}^{e} \\
\left(\AA^{3}\right)\end{array}$ & $\begin{array}{l}\Delta \alpha_{j}^{e} \\
\left(\AA^{3}\right)\end{array}$ \\
\hline 0 & 2.236 & & & 7.24 & 2.87 & $\alpha_{2}^{e}: 1.30$ & $\cdots$ & $\cdots$ \\
\hline 0.2 & 2.248 & & & 7.47 & 2.96 & $\alpha_{3}^{e}: 3.70$ & 0.09 & -0.56 \\
\hline 0.4 & 2.254 & & & 7.72 & 3.06 & $\alpha_{3}^{e}: 3.68$ & 0.19 & -0.58 \\
\hline 0.6 & 2.287 & & & 8.15 & 3.23 & $\alpha_{3}^{e}: 3.85$ & 0.36 & -0.41 \\
\hline \multirow{2}{*}{$\begin{array}{l}\text { Fused } \\
\text { silica }\end{array}$} & 2.13 & & & 7.47 & 2.96 & $\alpha_{1}^{e}: 2.96$ & & \\
\hline & & & & \multicolumn{5}{|c|}{ Ionic polarizability } \\
\hline $\mathrm{Al} / \mathrm{Na}$ & & & & $\begin{array}{c}P_{m}^{i} \\
\left(\mathrm{~cm}^{3}\right)\end{array}$ & $\begin{array}{c}\alpha_{t}^{i} \\
\left(\AA^{3}\right)\end{array}$ & $\begin{array}{c}\alpha_{\dot{j}}^{i} \\
\left(\AA^{3}\right)\end{array}$ & $\begin{array}{l}\Delta \alpha_{t}^{i} \\
\left(\AA^{3}\right)\end{array}$ & $\stackrel{\Delta \alpha_{j}^{i}}{\left(\AA^{3}\right)}$ \\
\hline 0 & & & & 9.57 & 3.79 & $\alpha_{2}^{i}: 4.19$ & $\cdots$ & $\cdots$ \\
\hline 0.2 & & & & 10.01 & 3.97 & $\alpha_{3}^{i}: 6.22$ & 0.18 & -0.23 \\
\hline 0.4 & & & & 10.60 & 4.20 & $\alpha_{3}^{i}: 6.41$ & 0.41 & -0.04 \\
\hline 0.6 & & & & 11.46 & 4.54 & $\alpha_{3}^{i}: 6.68$ & 0.75 & 0.23 \\
\hline $\begin{array}{l}\text { Fused } \\
\text { silica }\end{array}$ & & & & 5.71 & 2.26 & $\alpha_{1}^{\vec{i}}: 2.26$ & & \\
\hline
\end{tabular}

microscopic level. Classical Clausius-Mossotti equation relates dielectric constant $\epsilon$ to the polarizability $\alpha$ of these structural units and their number density, $N$ :

$$
\frac{\epsilon-1}{\epsilon+2}=\frac{4 \pi}{3} \sum_{j} N_{j} \alpha_{j}
$$

Equation (1) is in cgs units, and the summation is taken over various $j$ structural units. The disordered nature of glass structure satisfies the assumption of local field used in deriving the Clausius-Mossotti equation. The number density $N_{j}$ can be expressed as $N_{j}=N_{0} N_{\mathrm{jm}} / V_{m}$, where $N_{0}$ is the Avogadro's number, $N_{\mathrm{jm}}$ is the mole fraction of $j$ th structural unit, and $V_{m}$ is the molar volume. If the molar polarizability $P_{m}$ is defined as ${ }^{18}$

$$
P_{m} \equiv \frac{4 \pi}{3} N_{0} \sum_{j} N_{\mathrm{jm}} \alpha_{j}
$$

then the Clausius-Mossotti equation becomes

$$
\frac{\epsilon-1}{\epsilon+2}=\frac{P_{m}}{V_{m}} .
$$

The molar volume of the present SAS glasses was calculated using $V_{m}=M_{m} / \rho$ where $M_{m}$ is the molar weight, and $\rho$ is the density; the molar polarizability was calculated from Eq. (3) using observed $\epsilon$ and $V_{m}$. The results of these calculations are reported in Table II.

The mechanisms of dielectric polarization responsible for $\epsilon_{\text {i.f. }}$ include both the electronic polarization and the ionic polarization. The molar polarizability is, therefore, a sum of the electronic molar polarizability $P_{m}^{e}$, and the ionic molar polarizability $P_{m}^{i}$ i.e., $P_{m}=P_{m}^{e}+P_{m}^{i}$. The electronic molar polarizability can be calculated by the Clausius-Mossotti equivalent Lorentz-Lorenz equation for optical frequencies, by using optical frequency dielectric constant $\epsilon_{\infty}$ which is related to the index of refraction $n$ by the equation $\epsilon_{\infty}=n^{2}$. The ionic molar polarizability is, therefore, calculated by subtracting $P_{m}^{e}$ from $P_{m}$. The indices of refraction of the SAS glasses are taken from Ref. 19 with $n=1.495,1.499$, 1,502 , and 1.512 for $x=0,0.2,0.4$, and 0.6 glasses, respectively. The calculated values of $P_{m}^{e}$ and $P_{m}^{i}$ are shown in Table II.

For the discussion of polarizabilities at microscopic level, it is convenient to define a total molecular polarizability per mole as $\alpha_{t} \equiv \sum N_{\mathrm{jm}} \alpha_{j}$ which is then related to $P_{m}$ by $P_{m}=\left(4 \pi N_{0} / 3\right) \alpha_{t}$. The variation trend of $P_{m}$ and $\alpha_{t}$ is, therefore, the same because of the linear proportionality. Similarly, we define $P_{m}^{e}=\left(4 \pi N_{0} / 3\right) \alpha_{t}^{e} \quad$ and $P_{m}^{i}=\left(4 \pi N_{0} / 3\right) \alpha_{t}^{i}$, where $\alpha_{t}^{e}$ and $\alpha_{t}^{i}$ are the electronic and 
ionic molecular polarizability, respectively. Note that the polarizabilities are additive, i.e., $\alpha_{t}=\alpha_{t}^{e}+\alpha_{t}^{i}$. The calculated $\alpha_{t}$ 's, $\alpha_{t}^{e}$, s, and $\alpha_{t}^{i}$, s are also reported in Table II. The variation of the molar and the molecular polarizabilities with composition can be understood in terms of the variation of $N_{j}$ 's and $\alpha_{j}$ 's as discussed next.

The microscopic sources of dielectric polarization in the present SAS glasses can be conveniently considered by dividing the structure of glass into three parts: the silicon tetrahedra $\left[\mathrm{SiO}_{2}\right]$, the nonbridging oxygen-sodium ion pairs $\left[\mathrm{O}_{1 / 2}-\mathrm{Na}\right]$, and the aluminum tetrahedra-sodium ion units $\left[\mathrm{AlO}_{2}-\mathrm{Na}\right]$. The polarizabilities of these structural units are designated as $\alpha_{1}$ for $\left[\mathrm{SiO}_{2}\right], \alpha_{2}$ for $\left[\mathrm{O}_{1 / 2}-\mathrm{Na}\right]$, and $\alpha_{3}$ for $\left[\mathrm{AlO}_{2}-\mathrm{Na}\right]$. According to the composition formula of the SAS glasses, there are $3-2 x \mathrm{~mol}$ of $\left[\mathrm{SiO}_{2}\right], 2-2 x \mathrm{~mol}$ of $\left[\mathrm{O}_{1 / 2}-\mathrm{Na}\right]$, and $2 x \mathrm{~mol}$ of $\left[\mathrm{AlO}_{2}-\mathrm{Na}\right]$ per $4-x$ mole glass. Therefore,

$$
\alpha_{t}=\sum_{j} N_{\mathrm{jm}} \alpha_{j}=\frac{1}{4-x}\left[(3-2 x) \alpha_{1}+(2-2 x) \alpha_{2}+2 x \alpha_{3}\right] .
$$

There are, thus, two structural variables $\left(\alpha_{1}\right.$ and $\left.\alpha_{2}\right)$ for sodium trisilicate glass and three $\left(\alpha_{1}, \alpha_{2}\right.$, and $\left.\alpha_{3}\right)$ for the SAS glasses. Having only one experimentally obtained parameter $\alpha_{t}$, it is not possible to determine $\alpha_{j}$ 's independently for each glass composition.

So to evaluate the $\alpha_{j}$ 's, an assumption is made that the polarizabilities of structural units remain constant with respect to composition. Then $\alpha_{j}$ 's are determined as follows: first, $\alpha_{1}$ is calculated for fused silica since $\left[\mathrm{SiO}_{2}\right]$ is the only constituent structural unit; then, $\alpha_{2}$ is calculated for sodium trisilicate glass by using the value of $\alpha_{1}$; finally, $\alpha_{3}$ is calculated for SAS glasses by using the values of $\alpha_{1}$ and $\alpha_{2}$. It has been found that an error from using $\alpha_{1}$ obtained for fused silica for analyzing $\alpha_{2}$ for sodium trisilicate glass would only introduce a constant error in the absolute values of $\alpha_{3}$, but not in its relative values. Therefore, a variation of $\alpha_{3}$ with composition would indicate the possible variations of true $\alpha_{1}, \alpha_{2}$, or $\alpha_{3}$ with composition, and would verify the validity of our assumption that the polarizabilities of structural units in the SAS glasses are composition independent.

The electronic polarizabilities, $\alpha_{j}^{e}$, s, and the ionic polarizabilities, $\alpha_{j}^{i}$,s of the structural units can also be calculated by the same procedure as adopted above for the $\alpha_{j}$ 's. For the calculation of $\alpha_{1}, \epsilon_{\text {i.f. }}=3.8,{ }^{20} \epsilon_{\infty}=2.13 \quad(n=1.46),{ }^{21}$ and $\rho=2.20 \mathrm{~g} / \mathrm{cm}^{3}$ (Ref. 21) are used for fused silica. The results of this calculation given in Table II show that the ionic polarizability of the structural units in the SAS glasses varies significantly with composition, but their electronic polarizability remains almost constant. Therefore, the overall polarizability has a significant composition dependence mainly because of the variation of ionic polarizability. The calculated electronic polarizabilities of the structural units are close to the true values; whereas, the calculated ionic and overall polarizabilities only indicate the qualitative trend of the true polarizabilities.

From the above evaluation of the polarizabilities of the structural units, the effect of the substitution of $\mathrm{Al}$ for $\mathrm{Si}$ on molecular polarizability can be readily understood. In this
TABLE III. Electronic polarizability of oxygen ions $\alpha_{\mathrm{O}}^{e}$, in various structural units.

\begin{tabular}{cccc}
\hline \hline & \multicolumn{3}{c}{$\alpha_{\mathrm{O}}^{e}\left(\AA^{3}\right)$} \\
\cline { 2 - 4 } $\mathrm{A} 1 / \mathrm{Na}$ & $\mathrm{Si}-\mathrm{O}-\mathrm{Si}$ & $\mathrm{Si}-\mathrm{O}-\mathrm{Al}$ & $\mathrm{Si}-\mathrm{O}-\mathrm{Na}$ \\
\hline 0 & 1.48 & $\ldots$ & 2.59 \\
0.2 & 1.48 & 1.85 & 2.59 \\
0.4 & 1.48 & 1.84 & 2.59 \\
0.6 & 1.48 & 1.92 & 2.59 \\
\hline \hline
\end{tabular}

regard, a polarizability difference, $\Delta \alpha_{t}$, is defined as the difference between $\alpha_{t}$ for the SAS and sodium trisilicate glasses. Also, a polarizability difference of $\left[\mathrm{AlO}_{2}-\mathrm{Na}\right]$ and $\left[\mathrm{SiO}_{2}\right]+\left[\mathrm{O}_{1 / 2}-\mathrm{Na}\right]$ structural units is termed as $\Delta \alpha_{j}=\alpha_{3}-\left(\alpha_{1}+\alpha_{2}\right)$. It can be deduced from Eq. (4) that $\Delta \alpha_{t}=2 x \Delta \alpha_{j}$. Therefore, the variation of $\alpha_{t}$ with $x$ depends on the polarizability difference between $\left[\mathrm{AlO}_{2}-\mathrm{Na}\right]$ and $\left[\mathrm{SiO}_{2}\right]+\left[\mathrm{O}_{1 / 2}-\mathrm{Na}\right]$ as well as the extent of aluminum substitution. The calculated values of $\Delta \alpha_{t}$ and $\Delta \alpha_{j}$ are shown in Table II. It can be seen that the substitution of $\mathrm{Al}$ for $\mathrm{Si}$ in the SAS glasses results in a smaller electronic polarizability of $\left[\mathrm{AlO}_{2}-\mathrm{Na}\right]$ than that of $\left[\mathrm{SiO}_{2}\right]+\left[\mathrm{O}_{1 / 2}-\mathrm{Na}\right]$. The effect of this substitution on the ionic polarizability is, however, unclear because of the qualitative nature of the calculated $\alpha_{j}^{i}$, s.

\section{Correlation between dielectric constant and chemical structure}

\section{Electronic polarizability}

The electronic polarization arises from the shift of the center of the negative electron cloud in relation to the positive atom nucleus in an electric field. In the simplest case of a monatomic gas, the electronic polarizability of an atom is proportional to the volume of the atom. In solids also, the electronic polarizability generally increases with the size of the ion, but the dependence on composition is considerably more complicated by the redistribution of charge during bonding. In general, the density of electron charge is relatively more important for a negative ion since its outer electrons are less firmly bound than those of a positive one. Among the four ions, $(\mathrm{O}, \mathrm{Na}, \mathrm{Al}$, and $\mathrm{Si})$, in SAS glasses, oxygen being an anion has the largest electronic polarizability. ${ }^{22,23}$ In fact, its electronic polarizability is so much larger that, by comparison, the polarizability of the other three elements may be neglected. Therefore, we discuss the calculated electronic polarizability of the SAS glasses in terms of the polarizability of oxygen ions only.

As noted in the previous section, the electronic polarizability of the various structural units in SAS glasses remains essentially constant. It is, then, possible to calculate the electronic polarizability of the oxygen ions $\alpha_{O}^{e}$ by dividing the electronic polarizability of various units by the number of oxygen ions in each unit, viz: two oxygen atoms with $\mathrm{Si}-\mathrm{O}-\mathrm{Si}$ bond in a $\left[\mathrm{SiO}_{2}\right]$ unit; two oxygen atoms with $\mathrm{Si}-\mathrm{O}-\mathrm{Al}$ bond in a $\left[\mathrm{AlO}_{2}-\mathrm{Na}\right]$ unit; and one half of an oxygen ion with $\mathrm{Si}-\mathrm{O}-\mathrm{Na}$ bond in a $\left[\mathrm{O}_{1 / 2}-\mathrm{Na}\right]$ unit. The result of the calculation is shown in Table III. The polarizability for oxygen ions in $\mathrm{Si}-\mathrm{O}-\mathrm{Si}$ and $\mathrm{Si}-\mathrm{O}-\mathrm{Na}$ bonds is 


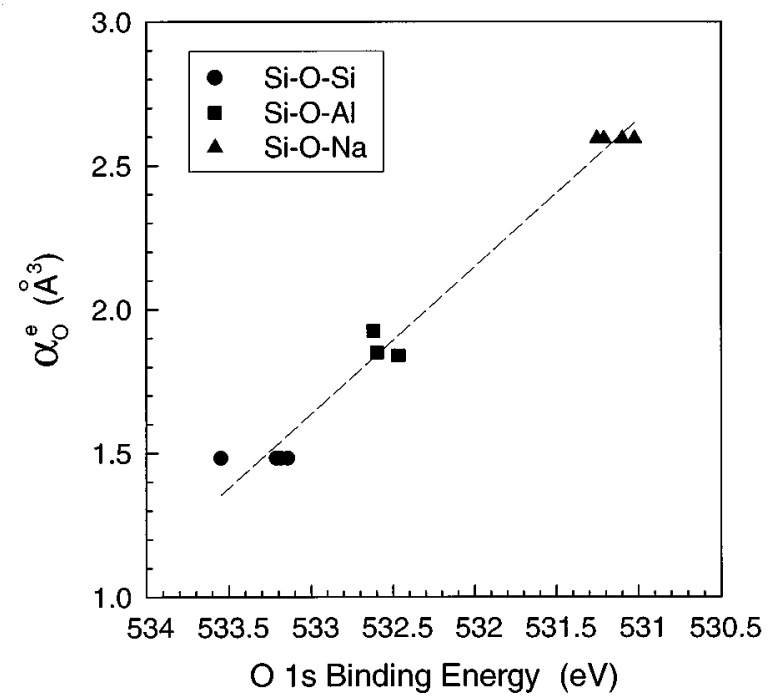

FIG. 8. Correlation between the electronic polarizability of oxygen ions and the O $1 s$ XPS binding energy in the SAS glasses.

independent of composition as assumed. All the possible variation of the electronic polarizability of oxygen ions is reflected in a small change of the polarizability of oxygen ions in $\mathrm{Si}-\mathrm{O}-\mathrm{Al}$. Nevertheless, there is a significant difference among the values for different units, specifically the electronic polarizability of oxygen ions increases in the order of $\mathrm{Si}-\mathrm{O}-\mathrm{Si}<\mathrm{Si}-\mathrm{O}-\mathrm{Al}<\mathrm{Si}-\mathrm{O}-\mathrm{Na}$.

Next we examine the relationship between polarizability and electron charge density around oxygen. According to Fig. 8, we find a simple correlation between the electronic polarizability of different types of oxygen ions and the $\mathrm{O} 1 \mathrm{~s}$ XPS binding energy plotted on a decreasing binding energy scale. A good linear relationship between the two parameters is given by the dashed straight line which is obtained by linear regression of all the data points in the plot. It was previously shown ${ }^{12}$ that the degree of negative charge on oxygen ions is inversely proportional to the $\mathrm{O} 1 s$ binding energy. Therefore, as the $\mathrm{O} 1 s$ binding energy decreases from $\mathrm{Si}-\mathrm{O}-\mathrm{Si}$ to $\mathrm{Si}-\mathrm{O}-\mathrm{Na}$, the degree of negative charge on oxygen ions increases. Consequently, Fig. 8 implies that the electronic polarizability of oxygen ions increases linearly with the degree of negative charge on the ions in the SAS glasses. We believe this is the first demonstration of a simple relationship between the electronic polarizability and an experimentally obtained charge density parameter.

\section{Ionic polarizability}

The ionic polarization arises from the displacement of positive and negative ions in relation to one another in an electric field. The ionic polarizability $\alpha_{i}$ of ion pairs can be described by the oscillator model as:

$$
\alpha^{i}=\frac{z^{2} e^{2}}{\left(2 \pi \nu_{0}\right)^{2} m},
$$

where $z e$ is the charge on an ion, $\nu_{0}$ is the lattice vibration frequency, and $m$ is the reduced mass. ${ }^{24,25}$ The denominator of Eq. (5) is simply the force constant of the bond between
TABLE IV. Ionic polarizability of sodium ions: $\alpha_{\mathrm{Na}}^{i}$ as calculated from dielectric measurements, and $\alpha_{\mathrm{Na}}^{i}{ }^{*}$ as determined theoretically assuming complete ionization of sodium ions. $\nu_{\text {eff }}$ is the effective vibration frequency of sodium ions.

\begin{tabular}{ccccc}
\hline \hline $\mathrm{A} 1 / \mathrm{Na}$ & $\alpha_{\mathrm{Na}}^{i}\left(\AA^{3}\right)$ & $\nu_{\mathrm{eff}}\left(\mathrm{cm}^{-1}\right)$ & $\alpha_{\mathrm{Na}}^{i} *\left(\AA^{3}\right)$ & $\left(\alpha_{\mathrm{Na}}^{i} / \alpha_{\mathrm{Na}}^{i}{ }^{*}\right)^{1 / 2}$ \\
\hline 0 & 4.14 & 173 & 5.68 & 0.854 \\
0.2 & 4.56 & 168 & 6.02 & 0.870 \\
0.4 & 5.05 & 165 & 6.24 & 0.899 \\
0.6 & 5.67 & 156 & 6.98 & 0.901 \\
\hline \hline
\end{tabular}

the ions. Clearly, the ionic polarizability is strongly affected by the chemical structure of the ions. The ionic polarizability of individual molecular units is more complicated to quantify, but it should depend on the bonding in a similar way. In SAS glasses, the ionic polarizability should include contribution from the vibrations within silicon and aluminum tetrahedra, and the vibration of sodium ions with respect to their charge compensating surroundings consisting of nonbridging oxygen ions and aluminum tetrahedra.

From the analysis of dielectric constant in Sec. IV B, the ionic polarizability of the structural units in SAS glasses is found varying significantly with composition. Since the silicon, aluminum, and oxygen XPS peak positions remain almost unchanged (see Sec. III A), the ionic polarizability of $\left[\mathrm{SiO}_{2}\right]$ and $\left[\mathrm{AlO}_{2}\right]$ units is assumed to be constant. The variation of ionic polarizability with composition is, therefore, attributed to the change of polarizability of sodium ions with respect to their surroundings. Furthermore, since a sodium ion is surrounded by a cage of several nonbridging oxygen ions and aluminum tetrahedra, and it has a well-defined chemical state, its vibrations with respect to surroundings can be considered as those of an oscillator made up of the sodium ion itself and the charge center of its cage. In this description, the ionic polarizability of sodium ions $\alpha_{\mathrm{Na}}^{i}$ can be considered as an average of $\left[\mathrm{O}_{1 / 2}-\mathrm{Na}\right]$ and $\left[\mathrm{AlO}_{2}-\mathrm{Na}\right]$ bonds according their relative concentration. That is, $\alpha_{\mathrm{Na}}^{i}$ $=\left[(2-2 x) \alpha_{2}^{i}+2 x \alpha_{3}^{i}\right] /[(2-2 x)+2 x]=(1-x) \alpha_{2}^{i}+x \alpha_{3}^{i}$ according to Eq. (4). One should note that the ionic polarizability of aluminum tetrahedra is inherently included in $\alpha_{3}^{i}$. It is not a part of the vibrations of sodium ions and is assumed to be constant. The calculated values of $\alpha_{\mathrm{Na}}^{i}$ are reported in Table IV which shows that the ionic polarizability of sodium ions increases with increasing concentration of aluminum in the SAS glasses.

If the description of vibration involving sodium ions is simplified by considering it as an oscillator, the ionic polarizability of sodium ions can be calculated from Eq. (5). In this calculation, $\nu_{0}$ is taken as the effective vibration frequency of sodium ions, $\nu_{\text {eff }}$, obtained from far-infrared spectra of the present glasses. ${ }^{26,27}$ The $m$ is taken to be the mass of a sodium ion since the mass of the site is considerably larger than that of a sodium ion. A directly measured value of $z$ is not available from experiments, so a value of $z=1$ is used assuming a total ionic character of sodium ions. The sodium ionic polarizability thus calculated is designated as $\alpha_{\mathrm{Na}}^{i}{ }^{*}$ with a superscript $*$ to indicate assumed complete ionization of sodium with $z=1$. The results of $\nu_{\mathrm{eff}}$ and $\alpha_{\mathrm{Na}}^{i}{ }^{*}$ are shown in Table IV. Since $\alpha_{\mathrm{Na}}^{i}$, which is obtained from the 


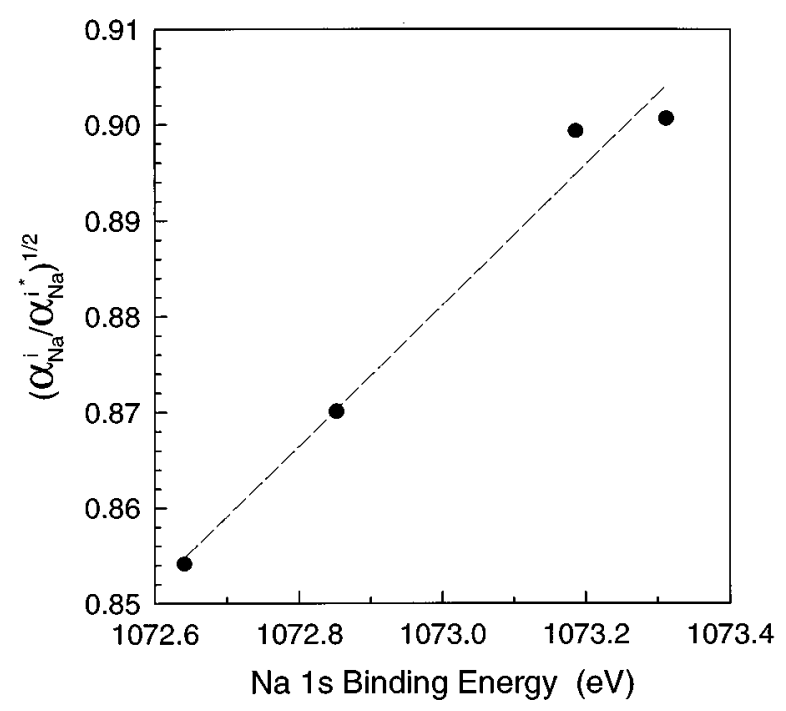

FIG. 9. Correlation between the ionic polarizability of sodium ions and the $\mathrm{Na} 1 s$ XPS binding energy in the SAS glasses.

dielectric constant analysis, contains possible deviation of $z$ from 1 because of the partially ionic characteristics of sodium ions, the square root of the ratio of experimental $\alpha_{\mathrm{Na}}^{i}$ to theoretical $\alpha_{\mathrm{Na}}^{i}{ }^{*}$ should be proportional to the actual value of $z$. Table IV shows that this ratio, $\left(\alpha_{\mathrm{Na}}^{i} / \alpha_{\mathrm{Na}}^{i}{ }^{*}\right)^{1 / 2}$, increases with increasing aluminum amount in the SAS glasses.

To establish a relation between ionic polarizability and the chemical structure of glass, $\left(\alpha_{\mathrm{Na}}^{i} / \alpha_{\mathrm{Na}}^{i}\right)^{1 / 2}$ is plotted against the Na $1 s$ XPS binding energy in Fig. 9. It shows that $\left(\alpha_{\mathrm{Na}}^{i} / \alpha_{\mathrm{Na}}^{i}{ }^{*}\right)^{1 / 2}$ increases linearly with $\mathrm{Na} 1 s$ binding energy. Since an increase of $\mathrm{Na} 1 s$ binding energy implies an increase of the positive charge on sodium ions, Fig. 9 suggests a parabolic dependence of $\alpha_{\mathrm{Na}}^{i}$ on the actual charge $z$ and reveals the importance of the experimentally determined chemical structure for determining the ionic polarizability.

\section{CONCLUSIONS}

Sodium ions have a well-defined chemical state in SAS glasses. They are only partially ionized in sodium trisilicate glass, and their ionicity increases when silicon is substituted by aluminum. A sodium ion is surrounded by several nonbridging oxygen ions and aluminum tetrahedra, and it is not possible to identify which sodium ion contributes the electron to a certain nonbridging oxygen ion or aluminum tetrahedron. The ionicity of silicon, aluminum, and oxygen atoms remains essentially unchanged in these SAS glasses.

The dielectric constant of the SAS glasses increases with increasing Al substitution. The electronic polarizability of constituent structural units remains constant with composition, while their ionic polarizability increases with increasing aluminum substitution.
The electronic polarizability of oxygen ions increases in the order of $\mathrm{Si}-\mathrm{O}-\mathrm{Si}<\mathrm{Si}-\mathrm{O}-\mathrm{Al}<\mathrm{Si}-\mathrm{O}-\mathrm{Na}$. It depends linearly on the negative charge on oxygen ions, and can be correlated to the $\mathrm{O} 1 s$ XPS binding energy. The ionic polarizability of sodium ions increases with increasing amount of aluminum in the SAS glasses. It correlates directly with the $\mathrm{Na} 1 s$ XPS binding energy.

\section{ACKNOWLEDGMENTS}

The authors wish to thank Dr. A. C. Miller for his help with the XPS experiments, and the National Science Foundation for financial support under Grant No. DMR-9225072. A grant from NATO (No. CRG931213) for international collaboration is also gratefully acknowledged.

${ }^{1}$ G. Geiger, Ceram. Bull. 69, 1131 (1990).

${ }^{2}$ R. R. Tummala, J. Am. Ceram. Soc. 74, 895 (1991).

${ }^{3}$ K. Kobayashi, J. Non-Cryst. Solids 109, 277 (1989).

${ }^{4}$ P. W. Bless, R. L. Wahlers, and S. J. Stein, in Glasses for Electronic Applications, edited by K. M. Nair (The American Ceramic Society, Westerville, OH, 1991), p. 397.

${ }^{5}$ G. M. Singer and M. Tomozawa, Phys. Chem. Glasses 30, 86 (1989).

${ }^{6}$ P. N. Kumta and M. A. Sriram, J. Mater. Sci. A 28, 1097 (1993).

${ }^{7}$ S. C. Cherukuri and S. K. Dey, in Glasses for Electronic Applications, edited by K. M. Nair (The American Ceramic Society, Westerville, OH, 1991), p. 355.

${ }^{8}$ S. V. J. Kenmuir, J. S. Thorp, and B. L. J. Kulesza, J. Mater. Sci. 18, 1725 (1983).

${ }^{9}$ R. N. Hampton, W. Hong, G. A. Saunders, and R. A. El-Mallawany, Phys. Chem. Glasses 29, 100 (1988).

${ }^{10}$ R. El-Mallawany, Mater. Chem. Phys. 37, 376 (1994).

${ }^{11}$ H. A. A. Sidek, I. T. Collier, R. N. Hampton, G. A. Saunders, and B. Bridge, Philos. Mag. B 59, 221 (1989).

${ }^{12}$ C. H. Hsieh, H. Jain, A. C. Miller, and E. I. Kamitsos, J. Non-Cryst. Solids 168, 247 (1994).

${ }^{13}$ A. K. Jonscher, Nature 267, 673 (1977).

${ }^{14}$ C. H. Hsieh and H. Jain, J. Non-Cryst. Solids 183, 1 (1995).

${ }^{15}$ D. A. McKeown, G. A. Waychunas, and G. E. Brown, Jr., J. Non-Cryst. Solids 74, 325 (1985)

${ }^{16}$ D. M. Zirl and S. H. Garofalini, J. Am. Ceram. Soc. 73, 2848 (1990).

${ }^{17}$ Y. Cao and A. N. Cormack, in Diffusion in Amorphous Materials, edited by H. Jain and D. Gupta (The Minerals, Metals \& Materials Society, Warrendale, PA, 1994), p. 137

${ }^{18}$ W. D. Kingery, H. K. Bowen, and D. R. Uhlmann, Introduction to Ceramics, 2nd ed. (Wiley, New York, 1976), Chap. 18.

${ }^{19}$ J. Schroeder, J. Non-Cryst. Solids 40, 549 (1980).

${ }^{20}$ R. N. Hampton, I. T. Collier, H. A. A. Sidek, and G. A. Saunders, J. Non-Cryst. Solids 110, 213 (1989).

${ }^{21}$ N. P. Bansal and R. H. Doremus, Handbook of Glass Properties (Academic, Orlando, FL, 1986), Chap 2.

${ }^{22}$ C. Kittel, Introduction to Solid State Physics, 6th ed. (Wiley, Singapore, 1986), Chap. 13

${ }^{23}$ H. Rawson, Properties and Applications of Glass (Elsevier, Amsterdam, The Neverlands, 1980), Chap. 6.

${ }^{24}$ G. G. Agrawal, H. P. Sharma, and J. Shanker, J. Phys. Chem. Solids 38, 815 (1977).

${ }^{25}$ A. K. Varshneya, Fundamentals of Inorganic Glasses (Academic, San Diego, CA 1994), Chap. 19.

${ }^{26}$ E. I. Kamitsos, J. A. Kapoutsis, H. Jain, and C. H. Hsieh, J. Non-Cryst. Solids 171, 31 (1994).

${ }^{27}$ E. I. Kamitsos, J. A. Kapoutsis, H. Jain, and C. H. Hsieh (to be published). 